\title{
Nondestructive identification of inhomogeneous residual stress state in deformable bodies on the basis of the acoustic sounding method
}

\author{
Vladimir Vladimirovich Dudarev ${ }^{\mathrm{a}}$, Rostislav Dmitrievich Nedin ${ }^{\mathrm{b}^{*}}$, \\ and Alexander Ovanesovich Vatulyan ${ }^{\mathrm{C}}$
}

Department of Theory of Elasticity, Faculty of Mathematics, Mechanics and Computer Sciences, Southern Federal University, 8a, Mil'chakova Street, 344090, Rostov-on-Don, Russia

adudarev_vv@mail.ru, brdn90@bk.ru, cvatulyan@math.rsu.ru

Keywords: Inhomogeneous residual stress, identification, nondestructive acoustical sounding, illposed inverse problems, nonlinear, iterative regularization, computational experiments, beam, plate, layer, tube

\begin{abstract}
Analysis of inhomogeneous residual stress (RS) fields in bodies is one of the major problems of the mechanics of deformable solid bodies. In the present research the new techniques of identification of inhomogeneous RS in bodies are developed on the basis of surface displacement measurement in a set of points under vibrating sounding load. Corresponding nonlinear ill-posed inverse problems (IP) are formulated and solved numerically by means of iterative regularization. Based on computational experiments, the most advantageous sounding load types and frequency ranges providing the best reconstruction accuracy are revealed. The examples for a cantilever, a plate, a layer, and a cylindrical tube are presented.
\end{abstract}

\section{Mathematical modeling of residual stress state (RSS)}

A linearized boundary problem on vibrations of a prestressed body has the following general form:

$$
\begin{aligned}
& T_{i j},_{j}+\rho b_{i}=\rho \frac{\partial^{2} u_{i}}{\partial t^{2}}, \quad T_{i j}=\Gamma_{i j}+\sigma_{i j}, \quad \sigma_{i j}^{0},_{j}+\rho b_{i}^{0}=0, \\
& \left.u_{i}^{0}\right|_{S_{u}^{0}}=f_{i}^{0},\left.\quad \sigma_{i j}^{0} n_{j}\right|_{S_{u}^{0}}=P_{i}^{0},\left.\quad u_{i}\right|_{S_{u}}=0,\left.\quad T_{i j} n_{j}\right|_{S_{u}}=P_{i},
\end{aligned}
$$

Here the notation $u_{i},{ }_{j}=\partial u_{i} / \partial x_{j}^{0}$ is used, where $x_{k}^{0}$ are coordinates of the body in its initial deformed configuration; $\rho$ is the body density; quantities with the superscript " 0 " are referred to the initial state, quantities without a superscript are the components of the corresponding incremental vectors and tensors: body force $\left(b_{i}\right)$, traction $\left(P_{i}\right)$, displacement $\left(u_{i}\right)$, the Cauchy stress $\left(\sigma_{i j}\right) ; T_{i j}$ are the components of nonsymmetrical incremental first Piola-Kirchhoff stress tensor.

The relations $\quad \Gamma_{i j}^{\mathrm{I}}=u_{i, m} \sigma_{m j}^{0}, \quad \Gamma_{i j}^{\mathrm{II}}=\frac{1}{4} \sigma_{m j}^{0}\left(3 u_{i, m}-u_{m, i}\right)-\frac{1}{4} \sigma_{m i}^{0}\left(u_{j, m}+u_{m, j}\right), \quad$ and $\Gamma_{i j}^{\mathrm{III}}=\sigma_{i j}^{0} u_{m, m}-\frac{1}{2} \sigma_{m j}^{0}\left(u_{m, i}-u_{i, m}\right)-\frac{1}{2} \sigma_{m i}^{0}\left(u_{j, m}+u_{m, j}\right)$ define three different forms of the constitutive relation for a prestressed body that are the most widespread at present; they were proposed by $\mathrm{E}$. Trefftz, M. Biot, C. Biezeno, H. Hencky and R. Southwell, and refined later by V.V. Novozhilov, L.M. Zubov, K. Washizu, L. Robertson, A. Hoger, C. Truesdell and others. In the present research, we shall use the RS model corresponding to the components $\Gamma_{i j}^{\mathrm{I}}$. 


\section{Problem on identification of RSS in beam and plate}

Refined statements of boundary problems on steady-state vibrations of different bodies in the framework of the plane stress state are derived. On the basis of the hypotheses $u_{1}=\theta x_{3}+\zeta, \quad u_{2}=0, \quad u_{3}=w$ (where $\theta=\theta\left(x_{1}\right)$ is the rotation angle of the beam principal axis due to the bending, $\zeta=\zeta\left(x_{1}\right)$ is the longitudinal displacement, $w=w\left(x_{1}\right)$ is the deflection), the following boundary problem

$$
\begin{aligned}
& {\left[\left(E_{2}+\Sigma_{11}^{2}\right) \theta^{\prime}+\left(E_{1}+\Sigma_{11}^{1}\right) \zeta^{\prime}+\Sigma_{13}^{1} \theta\right]^{\prime}-\mathrm{M} w^{\prime}-\Sigma_{13}^{1} \theta^{\prime}-\Sigma_{13}^{0} \zeta^{\prime}-\left(\mathrm{M}+\Sigma_{33}^{0}\right) \theta+\omega^{2}\left(\mathrm{P}_{1} \zeta+\mathrm{P}_{2} \theta\right)=0} \\
& {\left[\left(E_{1}+\Sigma_{11}^{1}\right) \theta^{\prime}+\left(E_{0}+\Sigma_{11}^{0}\right) \zeta^{\prime}+\Sigma_{13}^{0} \theta\right]^{\prime}+\omega^{2}\left(\mathrm{P}_{0} \zeta+\mathrm{P}_{1} \theta\right)=0, \quad\left[\left(\mathrm{M}+\Sigma_{11}^{0}\right) w^{\prime}+\mathrm{M} \theta\right]^{\prime}+\omega^{2} \mathrm{P}_{0} w=0} \\
& w(0)=0, \theta(0)=0, \zeta(0)=0, \quad\left[\left(E_{2}+\Sigma_{11}^{2}\right) \theta^{\prime}+\left(E_{1}+\Sigma_{11}^{1}\right) \zeta^{\prime}+\Sigma_{13}^{1} \theta\right](l)=0, \\
& {\left[\left(E_{1}+\Sigma_{11}^{1}\right) \theta^{\prime}+\left(E_{0}+\Sigma_{11}^{0}\right) \zeta^{\prime}+\Sigma_{13}^{0} \theta\right](l)=0,\left[\left(\mathrm{M}+\Sigma_{11}^{0}\right) w^{\prime}+\mathrm{M} \theta\right](l)=-P}
\end{aligned}
$$

is derived for a prestressed elastic isotropic cantilever in the framework of the Timoshenko model, where the following notations are used:

$$
\begin{aligned}
& \mathrm{M}=\int_{F} \mu d F, E_{0}=\int_{F} E d F, E_{1}=\int_{F} E x_{3} d F, E_{2}=\int_{F} E x_{3}^{2} d F, \quad \Sigma_{11}^{0}=\int_{F} \sigma_{11}^{0} d F, \Sigma_{11}^{1}=\int_{F} \sigma_{11}^{0} x_{3} d F, \Sigma_{11}^{2}=\int_{F} \sigma_{11}^{0} x_{3}^{2} d F, \\
& \Sigma_{13}^{0}=\int_{F} \sigma_{13}^{0} d F, \Sigma_{13}^{1}=\int_{F} \sigma_{13}^{0} x_{3} d F, \Sigma_{33}^{0}=\int_{F} \sigma_{33}^{0} d F, \mathrm{P}_{0}=\int_{F} \rho d F, \mathrm{P}_{1}=\int_{F} \rho x_{3} d F, \mathrm{P}_{2}=\int_{F} \rho x_{3}^{2} d F,
\end{aligned}
$$

which are the average characteristics of the corresponding functions depending on axial coordinate $x$. Here the most general case of inhomogeneity is considered for all the problem parameters including the volume distribution of RSS.

On the basis of the hypotheses $u_{1}=\theta_{1} x_{3}+\zeta_{1}, \quad u_{2}=\theta_{2} x_{3}+\zeta_{2}, \quad u_{3}=w$ (where $\theta_{\alpha}=\theta_{\alpha}\left(x_{\beta}\right)$ are the rotation angles of the normal along the axes $x_{\alpha}, \zeta_{\alpha}=\zeta_{\alpha}\left(x_{\beta}\right)$ are the displacements in plate's plane, $w=w\left(x_{\beta}\right)$ is the plate deflection, $\left.\alpha, \beta=1,2\right)$, the following boundary problem

$$
\begin{aligned}
& Q_{\alpha \beta, \beta}-S_{\alpha}+\omega^{2}\left(\mathrm{P}_{2} \theta_{\alpha}+\mathrm{P}_{1} \zeta_{\alpha}\right)=0, R_{\alpha \beta, \beta}+\omega^{2}\left(\mathrm{P}_{1} \theta_{\alpha}+\mathrm{P}_{0} \zeta_{\alpha}\right)=0, T_{\alpha, a}+\mathrm{P}_{0} \omega^{2} w=0, \\
& \left.w\right|_{l_{u}}=0,\left.\theta_{\alpha}\right|_{l_{u}}=0,\left.\zeta_{a}\right|_{l_{u}}=0,\left.\quad Q_{\alpha \beta} n_{\beta}\right|_{l_{\sigma}}=0,\left.R_{\alpha \beta} n_{\beta}\right|_{l_{\sigma}}=0,\left.T_{\alpha} n_{\alpha}\right|_{l_{\sigma}}=-P .
\end{aligned}
$$

is derived for a prestressed thin isotropic plate in the framework of the Timoshenko model in the general case of inhomogeneity for all the problem parameters; here we denote

$$
\begin{aligned}
& \Psi_{\alpha \beta}^{\gamma}=\delta_{\alpha \beta}\left(\Lambda_{\gamma} \theta_{m, m}+\Lambda_{\gamma-1} \zeta_{m, m}\right)+\mathrm{M}_{\gamma}\left(\theta_{\alpha, \beta}+\theta_{\beta, \alpha}\right)+\mathrm{M}_{\gamma-1}\left(\zeta_{\alpha, \beta}+\zeta_{\beta, \alpha}\right)+\Sigma_{m \beta}^{\gamma} \theta_{\alpha, m}+\Sigma_{m \beta}^{\gamma-1} \zeta_{\alpha, m}+\Sigma_{\beta 3}^{\gamma-1} \theta_{\alpha} \\
& Q_{\alpha \beta}=\Psi_{\alpha \beta}^{2}, R_{\alpha \beta}=\Psi_{\alpha \beta}^{1}, \Lambda_{p}=\int_{-h / 2}^{h / 2} \lambda x_{3}^{p} d x_{3}, \mathrm{M}_{p}=\int_{-h / 2}^{h / 2} \mu x_{3}^{p} d x_{3}, \mathrm{P}_{p}=\int_{-h / 2}^{h / 2} \rho x_{3}^{p} d x_{3}, \Sigma_{\alpha \beta}^{p}=\int_{-h / 2}^{h / 2} \sigma_{\alpha \beta}^{0} x_{3}^{p} d x_{3}, \\
& S_{\alpha}=\mathrm{M}_{0}\left(w,_{\alpha}+\theta_{\alpha}\right)+\Sigma_{m 3}^{1} \theta_{\alpha, m}+\Sigma_{m 3}^{0} \zeta_{\alpha, m}+\Sigma_{33}^{0} \theta_{\alpha}, \\
& T_{\alpha}=\mathrm{M}_{0}\left(w,_{\alpha}+\theta_{\alpha}\right)+\Sigma_{\alpha m}^{0} w,_{m}, \quad \alpha, \beta, \gamma, m=1,2, p=0,1,2 .
\end{aligned}
$$

Using the finite element method, a numerical solution of the boundary problems is obtained, its accuracy is estimated; the effect of RS magnitudes on frequency response functions (FRF) of area's points is investigated; a comparison of the Euler-Bernoulli model with the Timoshenko model for bending vibration of a rod and the Kirchhoff model with the Timoshenko model for bending vibration of a thin plate is made. 
The IP is to determine the components of the RS tensor when the periodic sounding load is applied to a free surface $S_{\sigma}$, and the displacement field $\left.\underline{f}\right|_{S_{\sigma}}=\left.\underline{u}\right|_{S_{\sigma}}$ is measured in a set of points for several frequencies $\omega_{k} \in\left[\omega_{-}, \omega_{+}\right](k=\overline{1, m})$. Within the limits of such formulation, the IP is nonlinear and ill-posed; to solve it, the iterative regularization technique was employed, based on the generalized reciprocity relation

$$
\int_{V} \sigma_{m j}^{0(n)} u_{i, j}^{(n-1)} u_{i, m}^{(n-1)} d V+\int_{S_{\sigma}} P_{i}\left(f_{i}-u_{i}^{(n-1)}\right) d S_{\sigma}=0
$$

obtained in the paper [2]. The Eq. (5) describes an iterative process; at every its step the direct problem is solved for current approximation of RS $\tilde{\sigma}_{i j}^{0}$, and the corresponding displacement and strain fields are calculated; after that, new corrections of the unknown RS functions $\sigma_{i j}^{0(n)}$ are calculated from the Eq. (5); at the end of the iteration, the current approximation is revised due to the correction calculated: $\tilde{\sigma}_{i j}^{0}=\sigma_{i j}^{0}+\sigma_{i j}^{0(n)}$ (here tilde corresponds to the approximation revised). Such an approach requires searching an initial approximation $\sigma_{i j}^{0(0)}$ that may be chosen in some narrow class, for instance, constant or linear functions.

Several one-dimensional IP on identification of uniaxial RSS are considered, all of them are reduced to an iterative process, based on the Eq. (5): problems on a strip-like plate under in-plane and out-of-plane vibration for the Kirchhoff model, and on a beam for the Timoshenko model under bending vibration are reduced to the Fredholm integral equations (FIE) of the $1^{\text {st }}$ kind

$$
\begin{gathered}
\int_{\Omega} \sigma_{11}^{0(n)}\left[\left(u_{1,1}^{(n-1)}\right)^{2}+\left(u_{2,1}^{(n-1)}\right)^{2}\right] d \Omega=\int_{l_{\sigma}} P_{\alpha}\left(u_{\alpha}^{(n-1)}-f_{\alpha}\right) d l_{\sigma}, \\
\int_{S} \sigma_{11}^{0(n)}\left[\frac{h^{3}}{12}\left(\left(w_{, 11}^{(n-1)}\right)^{2}+\left(w_{, 12}^{(n-1)}\right)^{2}\right)+h\left(w_{,_{1}}^{(n-1)}\right)^{2}\right] d S=\int_{l_{\sigma}} q\left(w^{(n-1)}-f\right) d S, \\
\int_{0}^{l} \sigma_{11}^{0(n)}\left(J\left[\theta^{(n-1)}\right]^{\prime 2}+F\left[w^{(n-1)}\right]^{\prime 2}\right) d x_{1}=P\left(w^{(n-1)}(l)-f(l)\right), \omega \in\left[\omega_{-}, \omega_{+}\right],
\end{gathered}
$$

respectively [1]. Note, that solving of FIE of the $1^{\text {st }}$ kind is an ill-posed problem; to solve it, the regularization method of A.N. Tikhonov was used.

Two-dimensional IP on identification of plane RSS in a plate under in-plane and out-ofplane vibration in the framework of the Timoshenko model are considered. Both reciprocity relations take form

$$
\int_{\Omega}\left[\sigma_{11}^{0(n)} K_{11}^{(n-1)}+\sigma_{22}^{0(n)} K_{22}^{(n-1)}+\sigma_{12}^{0(n)} K_{12}^{(n-1)}\right] d \Omega=F^{(n-1)}
$$

where in case of in-plane vibration the kernels and the right part of the integral equation have form

$$
\begin{aligned}
& K_{11}^{(n-1)}=\left(u_{1,1}^{(n-1)}\right)^{2}+\left(u_{2,1}^{(n-1)}\right)^{2}, \quad K_{22}^{(n-1)}=\left(u_{1,2}^{(n-1)}\right)^{2}+\left(u_{2,2}^{(n-1)}\right)^{2}, K_{12}^{(n-1)}=2\left(u_{1,1}^{(n-1)} u_{1,2}^{(n-1)}+u_{2,1}^{(n-1)} u_{2,2}^{(n-1)}\right), \\
& F^{(n-1)}=\int_{l_{\sigma}} P_{\alpha}\left(u_{\alpha}^{(n-1)}-f_{\alpha}\right) d l_{\sigma},
\end{aligned}
$$

and in case of out-of-plane vibration for the Timoshenko model they are defined as follows: 


$$
\begin{aligned}
& K_{11}^{(n-1)}=H\left[\left(\theta_{1,1}^{(n-1)}\right)^{2}+\left(\theta_{2,1}^{(n-1)}\right)^{2}\right]+h\left(w_{,_{1}}^{(n-1)}\right)^{2}, K_{22}^{(n-1)}=H\left[\left(\theta_{1,2}^{(n-1)}\right)^{2}+\left(\theta_{2,2}^{(n-1)}\right)^{2}\right]+h\left(w_{, 2}^{(n-1)}\right)^{2}, \\
& K_{12}^{(n-1)}=2\left[H\left(\theta_{1,1}^{(n-1)} \theta_{1,2}^{(n-1)}+\theta_{2,1}^{(n-1)} \theta_{2,2}^{(n-1)}\right)+h w_{,_{1}}^{(n-1)} w_{,_{2}}^{(n-1)}\right], F^{(n-1)}=-\int_{l_{\sigma}} P\left(w^{(n-1)}-f\right) d l_{\sigma}
\end{aligned}
$$

An approach to RSS reconstruction is based on a preliminary partition of a plate's area into a mesh of superelements $\left\{\Omega_{k}\right\}_{k=1}^{N}$, and setting the Airy stress functions $\Phi_{k} \mathrm{I}_{\Omega_{k}}\left(x_{1}, x_{2}\right)$ at each of them, so that the unknown RS corrections are expressed in terms of the Airy functions introduced. By representing the functions $\Phi_{k}$ as linear combinations of biharmonic polynomials [3], the Eq. (6) may be reduced to an ill-conditioned system of linear algebraic equations with respect to the unknown expansion coefficients of each function $\Phi_{k} \mathrm{I}_{\Omega_{k}}$; to solve it, the Tikhonov regularization method was used. After that, the reconstruction result found in a class of piecewise functions was smoothed by cubical spline-interpolation.

In all the viewed IP, initial approximation for the unknown RS functions was searched in a class of linear functions by a minimization of residual functional. The computational experiments on RSS reconstruction were conducted. The maximum relative error between the exact and reconstructed laws did not exceed $8 \%$ that demonstrates an effectiveness of the algorithm proposed. For a majority of the experiments conducted, the second frequency range turned out to be the most auspicious from the point of view of reconstruction accuracy, in comparison with the first and the third frequency ranges; under in-plane vibration of a plate, the tangential load applied to free plate's boundary is more efficient than the normal one.

\section{Problem on identification of RSS in annular region}

Consider a problem on radial oscillation of annular region $\left(0<r_{1} \leq r \leq r_{2}\right)$ under inhomogeneous RSS. Assume that the only nonzero components of the RS tensor in a cylindrical coordinate system are $\sigma_{r r}^{0}(r)$ and $\sigma_{\varphi \varphi}^{0}(r)$ satisfying equilibrium conditions without body force, i.e. $b_{i}^{0}=0$ in the third equation of (1). The vibration is caused by axisymmetric load applied to the outer boundary; the inner one is free from load. The dimensionless motion equation and the boundary conditions take form

$$
\begin{gathered}
(1+\tau g) U^{\prime \prime}+\left(\tau g^{\prime}+\frac{1+\tau g}{\xi}\right) U^{\prime}-\left(\frac{1+\tau g}{\xi^{2}}+\frac{\tau g^{\prime}}{\xi}-\kappa^{2}\right) U=0 \\
(1+\tau g) U^{\prime}+\left.k \frac{U}{\xi}\right|_{\xi=1}=p^{*},(1+\tau g) U^{\prime}+\left.k \frac{U}{\xi}\right|_{\xi=\xi_{0}}=0
\end{gathered}
$$

where $p^{*}=-p r_{2} /(\lambda+2 \mu), \kappa^{2}=\rho \omega^{2} r_{2}^{2} /(\lambda+2 \mu)$ is the dimensionless parameter characterizing vibration frequency, $\xi \in\left[\xi_{0}, 1\right]$ is the dimensionless radial coordinate, $\xi_{0}=r_{1} / r_{2}, k=\lambda /(\lambda+2 \mu)$, $g(\xi)=\sigma_{r r}^{0} / \max _{\xi \in\left[\xi_{0}, 1\right]}\left|\sigma_{r r}^{0}\right|$ is a function describing the law of variation of the component $\sigma_{r r}^{0}$, $\tau=\max _{\xi \in\left[\xi_{0}, 1\right]}\left|\sigma_{r r}^{0}\right| /(\lambda+2 \mu)$ is a parameter characterizing the level of RSS, $u(r)=r_{2} U(\xi)$ is the displacement function. The direct problem on determination of the function $U(\xi)$ when the function $g(\xi)$ is known is solved numerically by the shooting method using Maple. An influence of RSS on FRF is sufficient only in close vicinity of resonances and for higher values of natural frequencies. 
Two approaches were proposed to solve the IP on a reconstruction of inhomogeneous RSS. The first one is based on reducing the IP to solving a $1^{\text {st }}$ order differential equation with variable coefficients with regard to the unknown function $g(\xi)$. As additional information we assume the given values of the displacement function in a finite set of points $\xi_{i} \in\left[\xi_{0}, 1\right]$ for one fixed frequency. An analysis of the computational experiments conducted for a law corresponding to the RSS from the Lame problem revealed that such an approach is workable in the frequency range below the first resonant. It is worth noting that one cannot occur a recovering of the origin function with satisfactory accuracy in any other frequency range due to the fact that in some points the coefficient by the leading derivative of the function $g(\xi)$ becomes zero. Reconstruction accuracy is determined mainly by an accuracy of the $1^{\text {st }}$ and the $2^{\text {nd }}$ derivatives of the displacement function.

Within the second approach, the displacement $d(\kappa)=U(1, \kappa), \kappa \in\left[\kappa_{-}, \kappa_{+}\right]$is assumed to be known [4]. The IP solving is based on a constructing of an iterative process. With the help of the linearization method, an operator relation is obtained that binds together the sought-for function of correction to the radial RS component $g_{1}$ with the displacement function to measure:

$$
\int_{\xi_{0}}^{1} \tau g_{1}(\xi)\left(\xi U_{0}^{\prime 2}(\xi, \kappa)+U_{0}^{2}(\xi, \kappa) / \xi\right) d \xi+\int_{\xi_{0}}^{1} \tau g_{1}^{\prime}(\xi) U_{0}^{2}(\xi, \kappa) d \xi=p^{*}\left(U_{0}(1, \kappa)-d(\kappa)\right), \kappa \in\left[\kappa_{-}, \kappa_{+}\right]
$$

where $U_{0}(1, \kappa)$ is the displacement function calculated at previous step. The solving of this equation is realized numerically by means of discretization of integral operators, by using difference scheme of calculating derivatives of the unknown function, and by further employing of the Tikhonov regularization method.

In the framework of each approach, the computational experiments on recovering laws of variation of RSS are conducted; the accuracy of the IP solution shows an effectiveness of the scheme proposed; the practical advices on realizing the most effective reconstruction procedure are formulated.

\section{Problem on identification of RSS in orthotropic layer}

On the basis of the general problem statement (1)-(2), the problem on vibration of an orthotropic layer with inhomogeneous RSS field depending on transverse coordinate $x_{3}$ is formulated. Assume that the RS tensor components satisfy the equilibrium equations from (1) accounting body force. Vibration is caused by the load $p_{3}=p \delta\left(x_{1}\right)$ applied to the top layer boundary $x_{3}=h$. Using the Fourier transformation for longitudinal coordinate $x_{1}$, three auxiliary boundary problems with regard to integral displacement components are formulated in which the RS components are separated. A statement of the problem describing transverse vibration of a layer has the following form:

$$
\left(\left(C_{55}+\sigma_{33}^{0}\right) v^{\prime}\right)^{\prime}+\rho \omega^{2} v=0,\left(\left(C_{55}+\sigma_{33}^{0}\right) v^{\prime}\right)(h)=p, v(0)=0
$$

The direct problem on determination of values of average displacement characteristic is reduced to the FIE of the $2^{\text {nd }}$ kind:

$$
V(\xi)=\int_{0}^{1} K(\eta, \xi) V d \eta+\int_{0}^{\xi} p_{0} f^{-1}(\eta) d \eta, K(\eta, \xi)=\kappa^{2} \int_{0}^{\min (\eta, \xi)} f^{-1}(s) d s, f(\xi)=1+\tau \varphi(\xi)
$$


where $V(\xi)=v\left(x_{3}\right) / h, \tau=\max _{x_{3} \in[0, h]}\left|\sigma_{33}^{0}\right| / C_{55}, \varphi(\xi)=\sigma_{33}^{0} / \max _{x_{3} \in[0, h]}\left|\sigma_{33}^{0}\right|$ is a function of variation of the component $\sigma_{33}^{0}, \quad \kappa^{2}=\rho h^{2} \omega^{2} / C_{55}$ is a parameter characterizing vibration frequency, $p_{0}=p / C_{55}, \xi=x_{3} / h \in[0 ; 1]$. This FIE is solved numerically by means of the collocation method. The effect of RSS levels on FRF is analyzed, and the dependency of transverse resonances on the magnitude of $\tau$ is revealed.

The solving of the IP on determination of RSS variation law $\varphi(\xi)$ in an orthotropic layer under transverse oscillations is realized by means of the iterative regularization. On the basis of the linearization method, the FIE of the $1^{\text {st }}$ kind is built that bind together values of the unknown correction function $\varphi_{1}$ and the given displacement transform function $d(\kappa)=V(1, \kappa)$ at the top boundary of the layer for a given frequency:

$$
\int_{0}^{1} \tau \varphi_{1}(\xi) V_{0}^{\prime 2}(\xi, \kappa) d \xi=p_{0}\left(V_{0}(1, \kappa)-d(\kappa)\right), \kappa \in\left[\kappa_{1}, \kappa_{2}\right]
$$

where $V_{0}$ is a function calculated at previous step. A feature of the kernel of this integral equation is that it may vanish for some vibration forms. The Eq. (7) is solved by the Tikhonov regularization method. Various numerical experiments on reconstruction of monotonic and non-monotonic laws are carried out. The maximum relative error between the exact and reconstructed laws does not exceed 5\%. To analyze an influence of input data error on reconstruction accuracy, another series of experiments is also conducted. It is investigated that the proposed method of IP solving is workable form small noise level (less than $0.1 \%$ ) of the input data, and the reconstruction error increases in proportion to the error of the input data. Results of the experiments carried out demonstrate the effectiveness of the IP solving algorithm proposed.

The research is done with the support of grants of the Russian Foundation of Basic Research (project codes: 13-01-00196-A, 14-01-31393 mol_a).

\section{References}

[1] R.D. Nedin, A.O. Vatulyan, On the Reconstruction of Inhomogeneous Initial Stresses in Plates, in: H. Altenbach, V. Eremeyev (Eds.), Advanced Structured Materials. Shell-like Structures. Nonclassical Theories and Applications, Springer-Verlag, Berlin-Heidelberg, 2011, pp. 165-182.

[2] R.D. Nedin, A.O. Vatulyan, Inverse Problem of Non-homogeneous Residual Stress Identification in Thin Plates, Int. J. Solids Struct. 50 (2013) 2107-2114.

[3] R.D. Nedin, A.O. Vatulyan, Concerning one approach to the reconstruction of heterogeneous residual stress in plate, ZAMM: Z. angew. Math. Mech. V. 94, 1-2 (2014) 142-149.

[4] V.V. Dudarev, A.O. Vatulyan, On restoring of the pre-stressed state in elastic bodies, ZAMM: Z. angew. Math. Mech. V. 91, 6. (2011) 485-492 